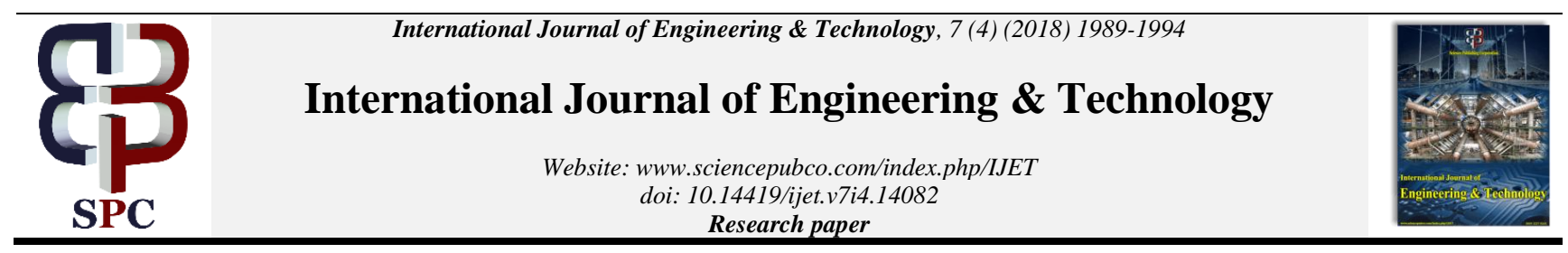

\title{
Preparation of insulating material based on rice straw and inexpensive polymers for different roofs
}

\author{
Reem Nasser ${ }^{1}$, M. A. $\operatorname{Radwan}^{1}$, M. A. Sadek ${ }^{1}$, Hany A. Elazab ${ }^{1 *}$ \\ ${ }^{1}$ Faculty of Engineering, Chemical Department, The British University in Egypt. \\ *Corresponding author E-mail:Hany.Elazab@bue.edu.eg
}

\begin{abstract}
Fossil fuel saving is part of the 21th century goal. One of the largest sectors that consumes energy is the building sector since it consumes $40 \%$ of the total energy in Europe through heating and cooling. Thus, the need of minimizing the energy consumption played an important role in the development of building insulation materials.

Selection of a proper material for building insulation differs according to the building surrounding climate and the properties required in the material. Building climate determines the insulating material location whether its internal or external while properties can differ according to many parameters that affect the material quality such as thermal insulation, acoustic insulation, waterproofing, resistance to fire and mechanical properties. Another factor is newly considered which doesn't affect the product quality but has an influence in the energy consumption is the environmental impact.

The aim of the research is considering most of the above factors through making a composite of rice straw particles bounded by a polymer to produce roof insulations. The utilization of wastes such as rice straw to produce a high quality product with the least price is the research main objective. The polymers selected for research are the unsaturated polyester resin and the polyurethane foam polymers. The polyester is not recently applied for insulation on comparison with the polyurethane foam which is dominant through the global market. At the end of the research, satisfying results have been achieved to nominate the $80 \%$ polyurethane foam and $20 \%$ rice straw composite as the best sample among all of the prepared samples. The other samples include polyester and rice straw composite with different compositions. The foam and straw sample have the best insulation with value of $0.1627 \mathrm{~W} / \mathrm{m} \mathrm{oC}$. The compression strength has also been improved on addition of rice straw to the polyurethane foam by three times the initial value. Also, the density measurement revealed a very light composite which is suitable for the construction field requirements. This results in reducing the cost by $28.35 \%$. On contrast, polyester samples which have very high density and higher $\mathrm{K}$ factor are not so efficient, so it is suggested that they can be applied at rural regions due to their low cost.
\end{abstract}

Keywords: Insulating Materials; Rice Straw; Polymers.

\section{Introduction}

The need behind the insulation material not only to provide comfort in buildings but to save Energy.[1-2]Since the majority of energy are consumed in the building sector through heating and cooling loads, so it was necessary to develop new materials that meet the requirements of different places with different climate conditions. It is also well established that nanotechnology and nanocomposites play an important role in enhancement of some features in this field.[3, 11-17]

Material selection is done based on a strategy beginning with deciding the best location for insulation material to be installed, and ends with the material characterization that depends on various parameters as thermal conductivity, acoustic insulation, waterproofing, resistance to fire, mechanical properties and finally the material environmental impact.[12, 18-25] Other factors are also considered in this strategy such as the availability and the cost. This enhanced the continuous seeking of new materials and the supporting of research institutions around the world to discover new materials with modified characteristics that can be available in the market with proper price.[26-32]

Some organizations currently classify the materials according to the market need and history into conventional and alternative ma- terials. For the time being, mineral wool and plastic foams are the most popular insulation materials with very few contributions from the natural materials. The main problem of most of the conventional materials is the high consumption of energy during their manufacturing process as well as being manufactured from petroleum extracted products which are non-renewable sources; this was the reason behind encouraging alternative materials especially natural materials.[2], [33-38], [42]

Environmental issues such as the global warming and the pollution has been lately forming a public issue. Concerning the global warming, it takes place due to the energy over consumption in industry and building sectors, this energy consumption increases the carbon dioxide concentration in the atmosphere which trap the heat and form the global warming. In addition, people get rid of some wastes as agricultural wastes through burning, this result in polluting the environment and harming different creatures.[39-43] Based on energy saving and environmental considerations, insulating composite of unsaturated polyester resin and rice straw is being investigated and tested. Rice straw is selected for many reasons such as its availability, low cost of purchasing, environmental protection from this burned waste and finally it has a low thermal conductivity. On the other hand, unsaturated polyester resin is selected due to its binding properties, low thermal conductivity, availability and its low cost. In addition, the currently ap- 
plied polyurethane foam will be further studied and a comparison will be made between different samples of both polymers.[2, 14]

The insulating materials used nowadays as polyurethane foam are expensive and imported from abroad while rice straw and used tires are cheap and available materials. These cheap materials are considered as undesirable and serious environmental problems occur to get rid of them. Applying these materials with inexpensive thermoset polymer could produce cheap national insulating tiles with very good mechanical, waterproofing, and thermal insulating characteristics.

The aim of this work is using unsaturated polyester as inexpensive polymer with small particle size rice straw to design and produce cheap insulating roof tiles which have excellent thermal insulation and mechanical characteristics as well as working as a waterproofing material.[13], [15], [17], [19]

\section{Experimental}

The chemicals were used as received without further treatment and they were purchased from Sigma Aldrich.

\subsection{Polyester/rice straw sample}

In typical synthesis of polyurethane foam, the used mold is cleaned properly, then adequate amounts from the Vaseline is added in order to facilitate the process of removing the polyurethane from the mold. Then, $70 \mathrm{~g}$ of polyester were added to $4-5 \%$ by weight of the initiator using a clean dropper. The mixture is then mixed properly using a stirring rod. Hence, $4-5 \%$ by weight of the activator was added using a clean dropper while mixing the mixture properly. Then, $30 \mathrm{~g}$ of grinded rice straw were added to the mixture and stirred vigorously until the mixture becomes homogeneous. The sample is then poured inside the mould and put the flat cover to make the sample surface uniform. To obtain better results, samples can be pressed using the hydraulic press at 100 bars. While small samples for compression and thermal conductivity tests can be poured in small porcelain cylindrical boxes with 50 mm diameter.

The sample is then left at the open air to dry for around 2-3 days until it completely cures. Finally, the plastic sheet is removed out of the mould. The previous procedures were also repeated with ratios of $3: 2$ and $1: 1$ between polyester resin and rice straw.

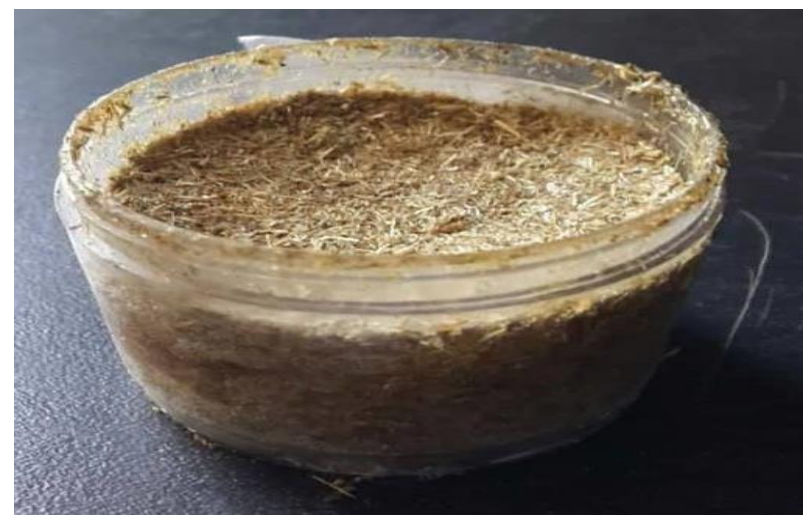

Fig. 1: Polyester/Rice Straw Sample for Testing.

\subsection{Preparation of polyurethane foam / rice straw sam- ple}

In the adopted procedure, the used mold is cleaned properly, and then adequate amounts of Vaseline were added to facilitate removing the polyurethane from the mold. The mould was covered with plastic bag to to keep it always clean and easily take the sample out of the mould. Then, the internal surface of the mould and the plastic sheet with any lubricating medium such as Vaseline. $50 \mathrm{ml}$ of diol were added inside an empty measuring cylinder then pour it in the mould. Then, $50 \mathrm{ml}$ of diisocyanate were also added in- side another clean measuring cylinder and pour it on the diol. Hence, the measuring cylinder was filled with grinded rice straw until it reaches $25 \mathrm{ml}$ then it was poured into the mixture. The mixture was mixed roughly using a stirring rod until the gas starts to evolve and sample expansion begins. The mixture was left for a day until it becomes totally cured and no heat is expelled. Then, the plastic sheet was removed out of the mould. Samples were cut based on the required dimensions.

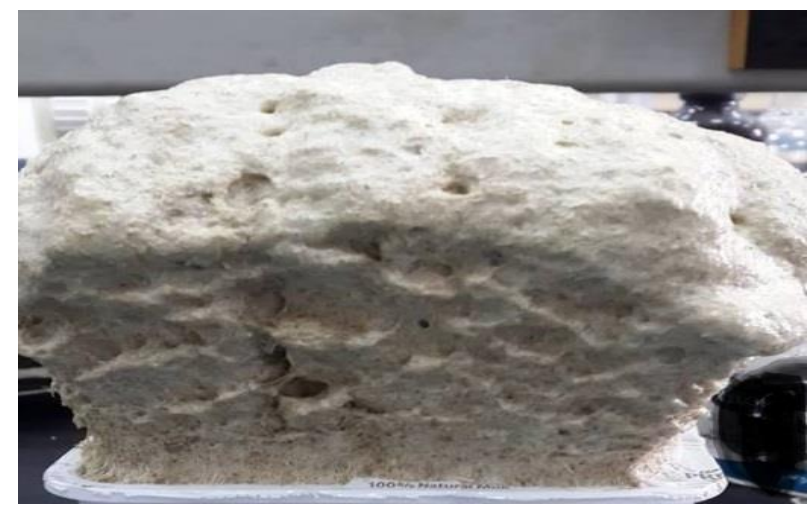

Fig. 2: Polyurethane Foam/ Rice Straw Sample.

\subsection{Preparation of pure polyester}

In typical procedure, small porcelain cylindrical boxes of $50 \mathrm{~mm}$ diameter were used. Those boxes were covered with lubricating medium such as Vaseline. $70 \mathrm{~g}$ of polyester was then added to 4-5 $\%$ by weight of the initiator using a clean dropper. The mixture was mixed properly using a stirring rod. Then, $2-2.5 \%$ by weight of the activator was added using a clean dropper and mix again the mixture properly. The mixture was then poured inside the boxes. The mixture was left on the open air around 2-3 days until it completely cures. Finally, the box was carefully removed and the sample was extracted out.

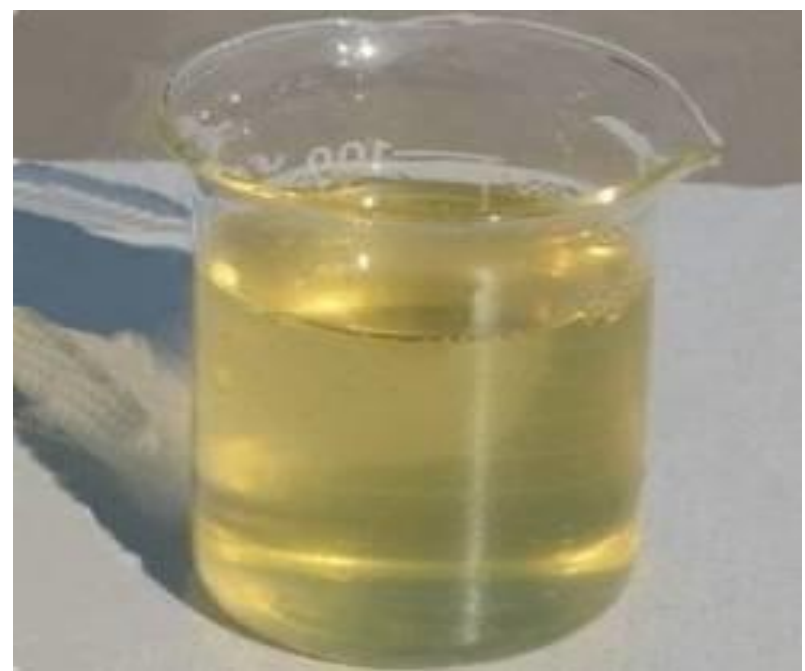

Fig. 3: Pure Polyester during Preparation.

\subsection{Preparation of pure polyurethane foam}

In the adopted procedure, the used mold is cleaned properly, and then adequate amounts of Vaseline were added to facilitate removing the polyurethane from the mold. The mould was covered with plastic bag to to keep it always clean and easily take the sample out of the mould. Then, the internal surface of the mould and the plastic sheet with any lubricating medium such as Vaseline. $50 \mathrm{ml}$ of diol were added inside an empty measuring cylinder then pour it in the mould. Then, $50 \mathrm{ml}$ of diisocyanate were also added inside another clean measuring cylinder and pour it on the diol. Hence, the measuring cylinder was filled with grinded rice straw until it reaches $25 \mathrm{ml}$ then it was poured into the mixture. The 
mixture was mixed roughly using a stirring rod until the gas starts to evolve and sample expansion begins. The mixture was left for a day until it becomes totally cured and no heat is expelled. Then, the plastic sheet was removed out of the mould. Samples were cut based on the required dimensions.

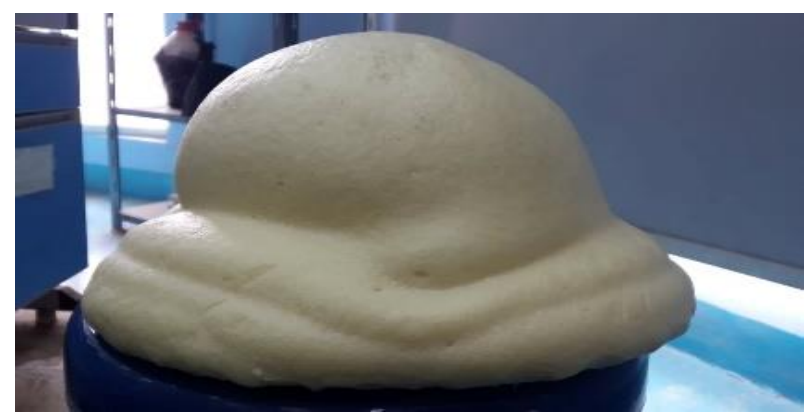

Fig. 4: Pure Polyurethane Foam Sample.

\subsection{Abrasion mould and sample}

The specimen dimension is $10 * 10 * 30 \mathrm{~mm}^{3}$.

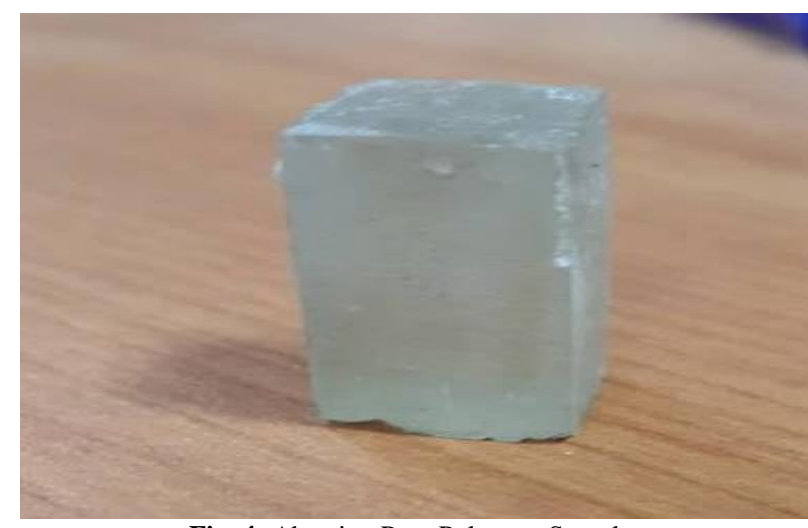

Fig. 4: Abrasion Pure Polyester Sample.

\subsection{Mechanical testing machine}

The tensile machine was used to measure the compression strength of the material and how it responds to the compression stresses. The compression machine has two jaws the first one is a fixed jaw and the second jaw is movable one that moves in a direction towards to the fixed jaw. The device is adjusted to compress the samples to half of its original length. The inputs to the machine test is the maximum load, the shape of the cross section of the samples (square, circular or tubal) and the dimensions of the specimen (length, height, diameter) according to the shape of the sample. The outputs are compression strength and the maximum elongation. The specimen of the polyurethane foam has square cross section while the samples of the polyester has circle cross section. The following two pictures showing the differences between some of the samples before and after compression test.
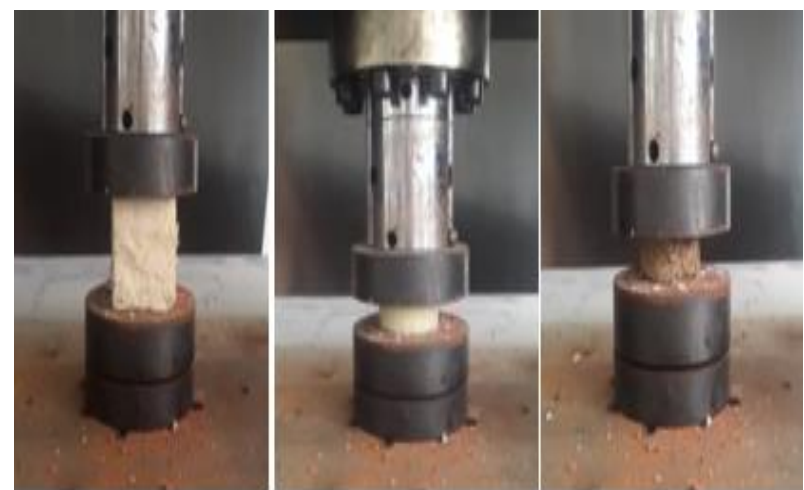

Fig. 5: Sample before and Compression.

\subsection{Thermal insulation measuring device}

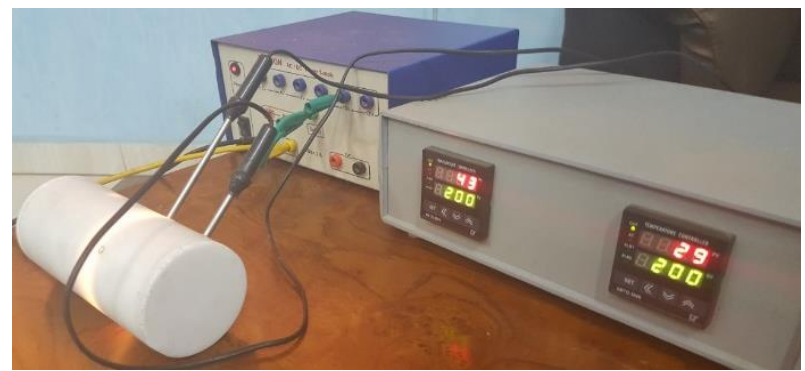

Fig. 6: Thermal Insulation Measuring Device.

The used thermal insulation measurement device composed of four main parts the first part is the power supply, the second part is the thermocouples that measures the temperature, the third part is the testing part where the specimen is applied and subjected to the heat, this art is insulated using a $0.5 \mathrm{~cm}$ Teflon to prevent heat losses to the surrounding. The fourth part is the digital thermometer reading. The supply power which generated the electrical heat with different voltages from 2 to 12 volt ( 2 volt, 4 volt, 6 volt, 8 volt, 10 volt and 12 volt) and different corresponding ampere leading to different amount of electrical energy. This circuit has a torch that has electrical energy equals to volt multiplied by the corresponding ampere. Assuming no loses all these amounts of energy is converted to heat energy using the torch. The torch was used instead of the heater to simulate the effect of the sun light that the insulation tile will be subjected to. The specimen must have circle cross section with dimension $50 \mathrm{~mm}$ for the diameter and a height of $0.5 \mathrm{~cm}$. hence, the thermal insulation factor $(\mathrm{K})$ is obtained by equalizing the two equations,

$\mathrm{Q}($ watt $)=\mathrm{V}($ volt $) * \mathrm{I}($ ampere $)=\frac{K A(T 2-T 1)}{x}$

where, $\mathrm{A}$ is the cross sectional area of the sample in $\mathrm{m}^{2}, \mathrm{~K}$ is the thermal conductivity in $\mathrm{W} / \mathrm{m} .{ }^{\circ} \mathrm{C}, \mathrm{T}_{2}$ is the temperature before the insulation in ${ }^{\circ} \mathrm{C}$ and the $\mathrm{T}_{1}$ is the temperature after the insulation in ${ }^{\circ} \mathrm{C}$ and $\mathrm{x}$ is the thickness of the sample in $\mathrm{m}$.

\subsection{Abrasion test device}

The used abrasion test mechanism is based on applying load that pushes the tested material to the moving steel wheel that is moving with 50 revolutions per minutes that is connected with a motor through a gear. The test is measuring the amount loss from the material under these conditions. The specimen dimension is from three to ten $\mathrm{cm}$ height and has square cross section of one $\mathrm{cm}$ length and one $\mathrm{cm}$ wide. The weight of the specimen is measured by the balance before and after the test in order to get the percent loss of the material after different times. The load is changed from two to five kilo grams.

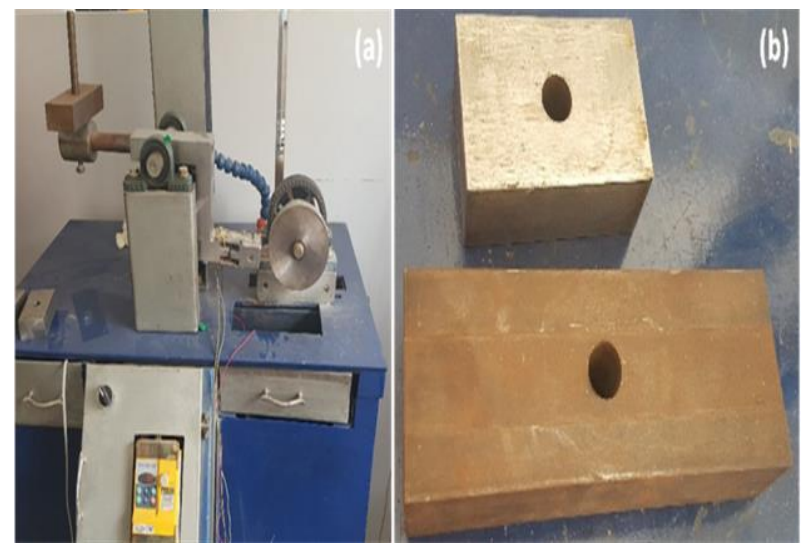

Fig. 7: A) Abrasion Machine, B) Abrasion Machine Loads. 


\section{Results and discussion}

\subsection{Thermal insulation calculation ( $\mathrm{K}$ factor)}

Thermal insulation is important as it indicates the ability of the material to prevent or reduce the heat transfer through the insulating material or between thermally contacted objectives. $\mathrm{K}$ factor that measures the ability of any materials to conduct the heat, thus, it is called the thermal conductivity factor. All the thermal conductive materials like metals have high value of thermal conductivity while the insulating materials have a thermal conductivity value that is lower than one. The lower the $\mathrm{K}$ value is, the more efficient the insulating material is.

From the experimental work done as shown in Table 1.

Table 1: Experimental Results Comparison Before and after Insulation

\begin{tabular}{ll}
\hline Compound name & $\mathrm{K}$ factor $\left(\mathrm{W} / \mathrm{m}^{\circ} \mathrm{C}\right)$ \\
\hline Pure polyurethane foam & 0.1850 \\
$80 \%$ polyurethane foam 20\% straw & 01627 \\
Pure polyester & 0.5898 \\
$70 \%$ polyester 30\% rice straw & 0.3774 \\
$60 \%$ polyester 40\% rice straw & 0.3254 \\
\hline
\end{tabular}

$\mathrm{Q}($ watt $)=\mathrm{V}($ volt $) * \mathrm{I}($ ampere $)=\frac{K A(T 2-T 1)}{X}$

Where, $\mathrm{A}$ is the cross sectional area of the sample in $\mathrm{m}^{2}, \mathrm{~K}$ is the thermal conductivity in $\mathrm{W} / \mathrm{m} .{ }^{\circ} \mathrm{C}, \mathrm{T}_{2}$ is the temperature before the insulation in ${ }^{\circ} \mathrm{C}$ and the $\mathrm{T}_{1}$ is the temperature after the insulation in ${ }^{\circ} \mathrm{C}$ and $\mathrm{x}$ is the thickness of the sample in $\mathrm{m}$.

The entire sample has, Diameter $=50 \mathrm{~mm}=0.05 \mathrm{~m}$, Length $=0.5$ $=\mathrm{cm}=0.005 \mathrm{~m}, \mathrm{I}=0.95 \mathrm{Amp}, \mathrm{V}=3.92$ Volt, $\mathrm{Q}=3.724$ Watt, $\mathrm{A}$ $=(\pi / 4) \mathrm{D}^{2}=0.001963495 \mathrm{~m}^{2}$

\subsection{Compression test results}

On comparing between the polyurethane foam two samples, it can be clearly observed that rice straw not only improved the insulation properties, but it also improved the mechanical properties. The compression test shows that the addition of rice straw has raised the exerted stress to nearly 2.9 times the initial value; at $50 \%$ deformation, the stress exerted corresponding to the applied load for the pure polyurethane foam was $61 \mathrm{KPa}$ while that for the polyurethane foam with rice straw filling was $175.5 \mathrm{KPa}$. This can be explained that the rice straw fill some of the low density voids in the low density polyurethane foam resulting in improving its strength. It is also worth mentioning that none of the samples have experienced any failures.

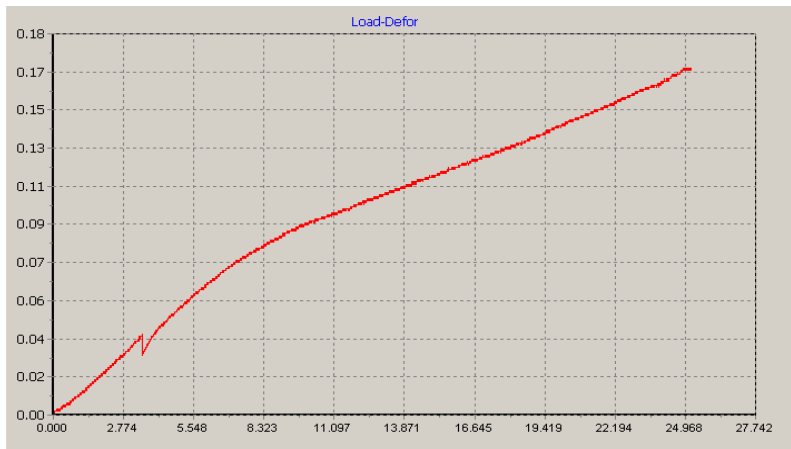

Fig. 8: Load Deformation Curve for 100\% Polyurethane Foam.

Concerning the three polyester samples, the case is totally different than that of the polyurethane foam samples. Increasing the amount of rice straw filling came up with negative effect for the mechanical properties. The pure polyester alone displayed a good result since it reached the maximum load of the tensile machine which is $100 \mathrm{KN}$ without experiencing any failure. However, the addition of $30 \%$ of rice straw as a filler decreased the exerted stress to nearly 2.1 times the initial value; this is because the new sample gained some elasticity.

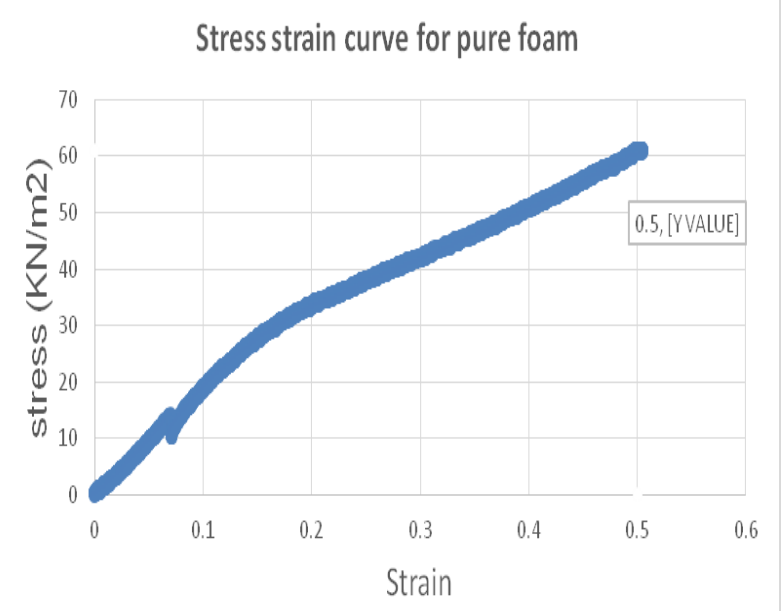

Fig. 9: Stress Strain Curve for 100\% Polyurethane Foam.

Also, it is observed that with compression, the sample responds by extending along its horizontal axis. With respect to the other sample, it was so brittle that it is easily compressed by very small loads; this can be illustrated as sample might didn't completely cure from inside and also the sample wasn't uniform enough due to the high percentage of rice straw.

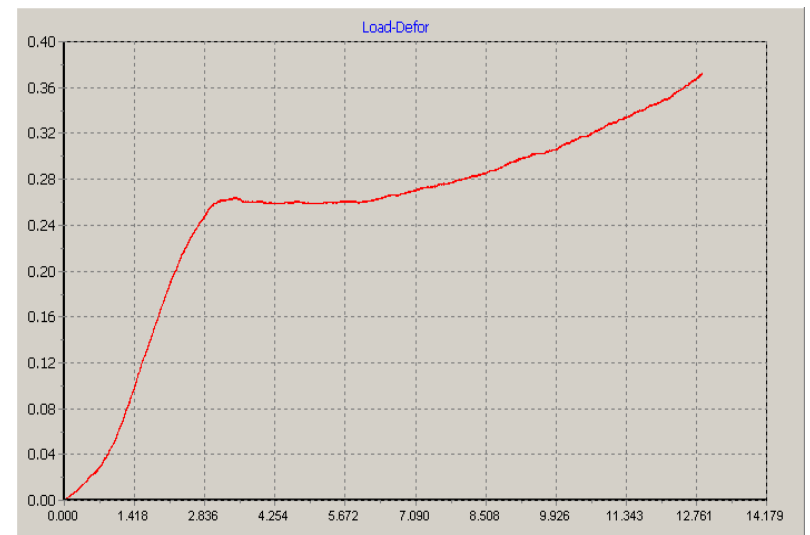

Fig. 10: Load Deformation Curve for $80 \%$ Polyurethane Foam 20\% Rice Straw.

It is expected that with samples compression before curing and better mixing methods between the polyester and the rice straw, the mechanical properties might highly improve. To sum up, the pure polyester sample is the best among the five samples as it reached the maximum load with $25 \%$ deformation and with no failures.

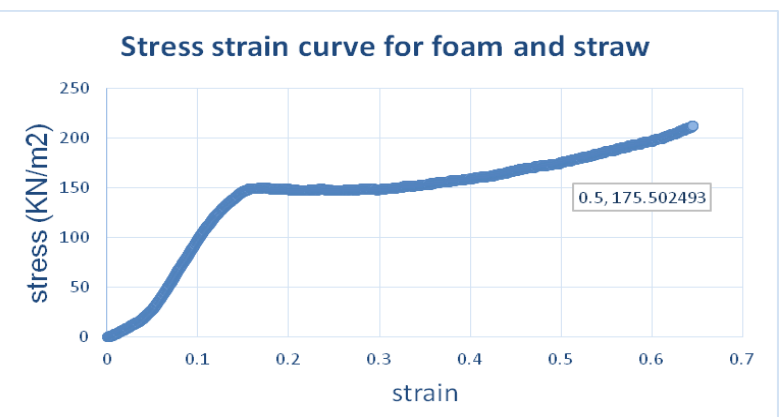

Fig. 11: Stress Strain Curve for 80\% Foam 20\% Rice Straw.

At $50 \%$ deformation, the exerted stress for the polyurethane foam and rice straw sample is $175.5 \mathrm{KPa}$. 


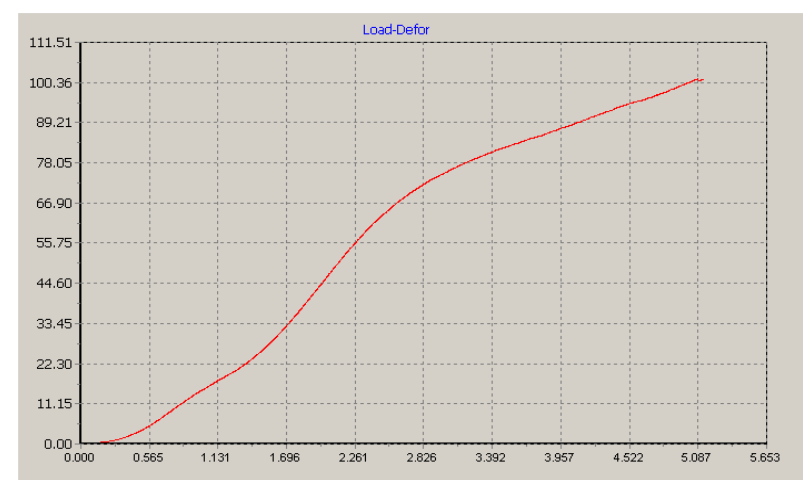

Fig. 12: Load Deformation Curve for 100\% Polyester.

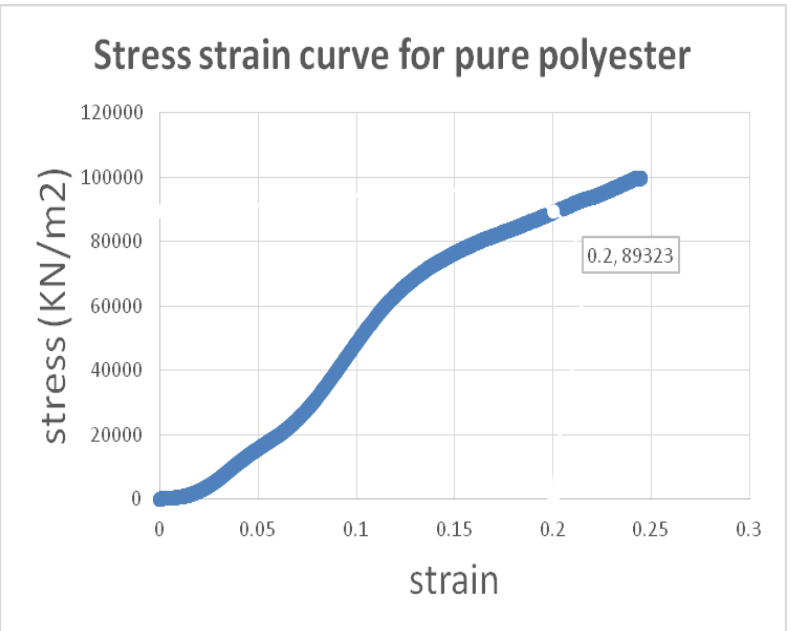

Fig. 13: Stress Strain Curve for Pure Polyester.

At $20 \%$ deformation, the exerted stress for the pure polyester sample is $89323 \mathrm{KPa}$. Also, the pure polyester is very rigid that it reached the maximum load of the universal testing machine which is $100 \mathrm{KN}$.

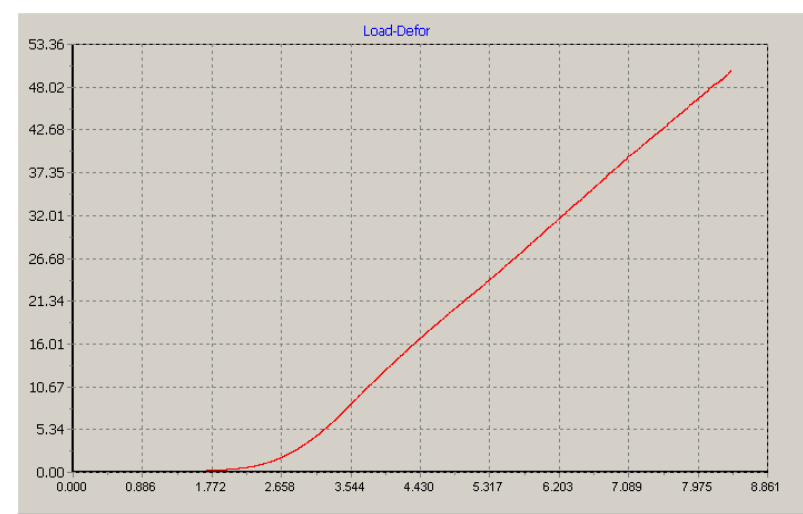

Fig. 14: Load Deformation Curve for 70\% Polyester 30\% Straw.

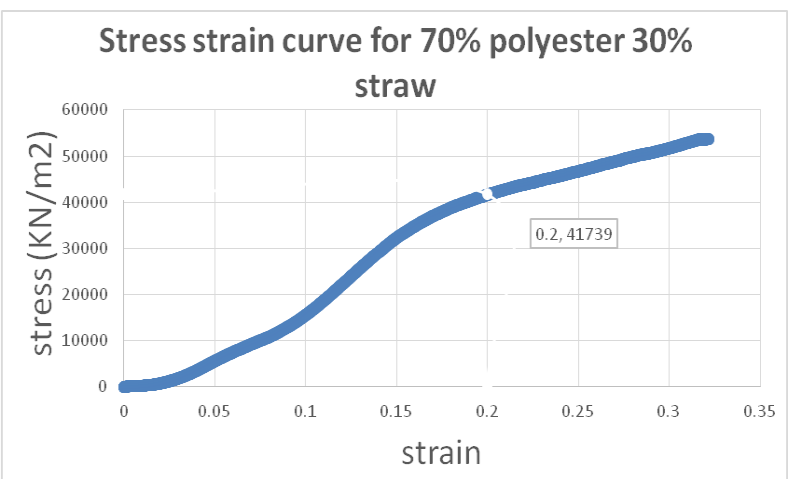

Fig. 15: Stress Strain Curve for $70 \%$ Polyester 30\% Straw.
At $20 \%$ deformation, the exerted stress for the $70 \%$ polyester and $30 \%$ straw sample is $41739 \mathrm{KPa}$.

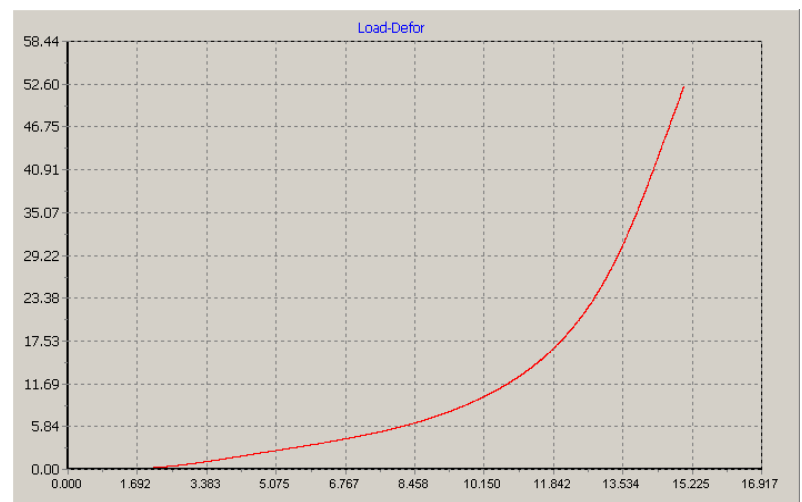

Fig. 16: Load Deformation Curve for 60\% Polyester 40\% Rice Straw.

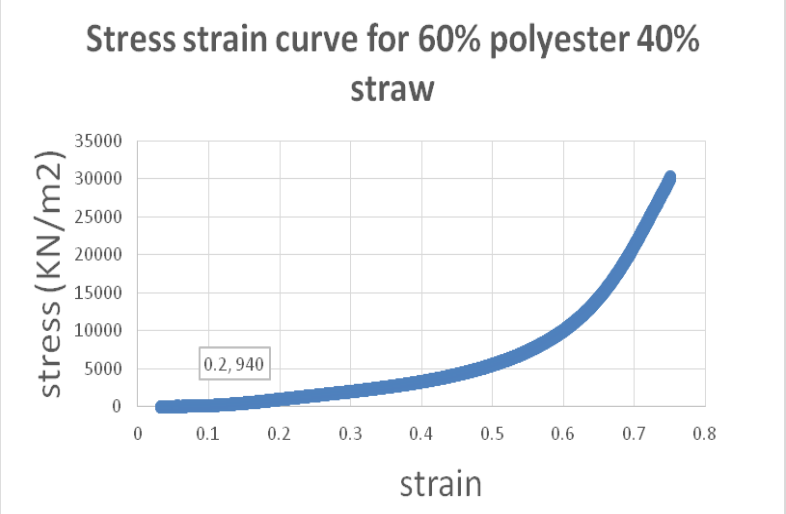

Fig. 17: Stress Strain Curve for 60\% Polyester 40\% Straw.

At $20 \%$ deformation, the exerted stress for the $60 \%$ polyester and $40 \%$ straw sample is $940 \mathrm{KPa}$.

\section{Conclusions}

In conclusion, the research investigation has achieved a new result through introducing an insulating material with lower thermal conductivity and lower cost. The polyurethane foam and rice straw composite proved to have a higher efficiency than the pure polyurethane foam through the insulation properties, mechanical properties and the overall cost. The thermal conductivity of this new composite is $12 \%$ lower than that of the polyurethane foam with thickness reduction of also $12 \%$. The advantage of this sample is also using a waste such as rice straw which is easily available for free. Rice straw is a good selection as a filler due to its low thermal conductivity which improves the material insulating properties. Consequently, this results in reducing the cost of insulation by $28.35 \%$. In spite of the cheap price of the polyester, but with the complicated technologies required to apply it to a large scale, the overall price would exceed the economical price, this is because the tiles would be manufactured at molds not sprayed like the polyurethane foam.

Thus, polyester and rice straw composite could be applied at small scale at rural regions where small areas are only needed to be insulated and no skyscrapers or high buildings that require reduction of loads.

\section{References}

[1] Barber, F.A. and M.A. Herbert, All-Suture Anchors: Biomechanical Analysis of Pullout Strength, Displacement, and Failure Mode. Arthroscopy: The Journal of Arthroscopic \& Related Surgery. 33(6): p. 1113-1121. 
[2] Dowson, M., et al., Predicted and in situ performance of a solar air collector incorporating a translucent granular aerogel cover. Energy and Buildings.49 p. 173-187.

[3] Elazab, H., et al., Microwave-assisted synthesis of Pd nanoparticles supported on $\mathrm{FeO}, \mathrm{CoO}$, and $\mathrm{Ni}(\mathrm{OH})$ nanoplates and catalysis application for CO oxidation. Journal of Nanoparticle Research, 2014. 16(7): p. 1-11.

[4] Elazab, H., et al., The Effect of Graphene on Catalytic Performance of Palladium Nanoparticles Decorated with $\mathrm{FeO}, \mathrm{CoO}$, and $\mathrm{Ni}$ (OH): Potential Efficient Catalysts Used for Suzuki CrossCoupling. Catalysis Letters. 147(6): p. 1510-1522.

[5] Elazab, H.A., et al., The continuous synthesis of Pd supported on Fe3O4 nanoparticles: A highly effective and magnetic catalyst for CO oxidation. Green Processing and Synthesis. 6(4): p. 413-424.

[6] Elazab, H.A., M.A. Sadek, and T.T. El-Idreesy, Microwaveassisted synthesis of palladium nanoparticles supported on copper oxide in aqueous medium as an efficient catalyst for Suzuki crosscoupling reaction. Adsorption Science \& Technology. 0 (0) p. 0263617418771777.

[7] Elazab, H.A., et al., Highly efficient and magnetically recyclable graphene-supported $\mathrm{Pd} / \mathrm{Fe} 3 \mathrm{O} 4$ nanoparticle catalysts for Suzuki and Heck cross-coupling reactions. Applied Catalysis A: General 2015.491: p. 58-69. https://doi.org/10.1016/j.apcata.2014.11.033.

[8] Mankarious, R.A., et al., Bulletproof vests/shields prepared from composite material based on strong polyamide fibers and epoxy resin. Journal of Engineering and Applied Sciences.12 (10): p. 2697-2701.

[9] Mohsen, W., M.A. Sadek, and H.A. Elazab, Green synthesis of copper oxide nanoparticles in aqueous medium as a potential efficient catalyst for catalysis applications. International Journal of Applied Engineering Research.12 (24): p. 14927-14930.

[10] Mostafa, A.R., H.A.-S. Omar, and A.E. Hany, Preparation of Hydrogel Based on Acryl Amide and Investigation of Different Factors Affecting Rate and Amount of Absorbed Water. Agricultural Sciences. Vol.08No.02: p. 11

[11] Radwan, M.A., et al., Mechanical characteristics for different composite materials based on commercial epoxy resins and different fillers. Journal of Engineering and Applied Sciences. 12(5): p. 1179-1185.

[12] Lauri, J. and R. Scapellato, Topics in Graph Automorphisms and Reconstruction. London Mathematical Society Lecture Note Series, Cambridge: Cambridge University Press.

[13] Naskar, A.K., A.K. Mukherjee, and R. Mukhopadhyay, Studies on tyre cords: degradation of polyester due to fatigue. Polymer Degradation and Stability, 2004. 83(1): p. 173-180. https://doi.org/10.1016/S0141-3910(03)00260-X.

[14] Papadopoulos, A.M., State of the art in thermal insulation materials and aims for future developments. Energy and Buildings, 2005. 37(1): p. 77-86. https://doi.org/10.1016/j.enbuild.2004.05.006

[15] Parres, F., J.E. Crespo-Amor $\widetilde{A}^{3}$ s, and A. Nadal-Gisbert, Mechanical properties analysis of plaster reinforced with fiber and microfiber obtained from shredded tires. Construction and Building Materials, 2009.23 (10): p. 3182-3188.

[16] Piszczyk, A.u., et al., Polyurethane/ground tire rubber composite foams based on polyglycerol: Processing, mechanical and thermal properties. Journal of Reinforced Plastics and Composites. 34(9): p. 708-717.

[17] Sarah, J., et al., Failure mechanism of the all-polyethylene glenoid implant. Journal of Biomechanics. 43(4): p. 714-719.

[18] Tang, Y., et al., Industrial polymers classification using laserinduced breakdown spectroscopy combined with self-organizing maps and K-means algorithm. Optik. 165: p. 179-185.

[19] Bennoud, Salim, and Mourad Zergoug. "Evaluation and Quantification of Electromagnetic Field Distribution for Different Configurations of Aeronautical Materials." Review of Industrial Engineering Letters 3, no. 2 (2016): 29-37.

[20] Elazab, H., et al., Microwave-assisted synthesis of Pd nanoparticles supported on $\mathrm{FeO}, \mathrm{CoO}$, and $\mathrm{Ni}(\mathrm{OH})$ nanoplates and catalysis application for CO oxidation. Journal of Nanoparticle Research, 2014. 16(7): p. 1-11.

[21] Elazab, H., et al., The Effect of Graphene on Catalytic Performance of Palladium Nanoparticles Decorated with $\mathrm{FeO}, \mathrm{CoO}$, and $\mathrm{Ni}$ $(\mathrm{OH})$ : Potential Efficient Catalysts Used for Suzuki CrossCoupling. Catalysis Letters. 147(6): p. 1510-1522.

[22] Elazab, H.A., et al., The continuous synthesis of Pd supported on $\mathrm{Fe} 3 \mathrm{O} 4$ nanoparticles: A highly effective and magnetic catalyst for CO oxidation. Green Processing and Synthesis. 6(4): p. 413-424.

[23] Elazab, H.A., et al., Highly efficient and magnetically recyclable graphene-supported $\mathrm{Pd} / \mathrm{Fe} 3 \mathrm{O} 4$ nanoparticle catalysts for Suzuki and Heck cross-coupling reactions. Applied Catalysis A: General, 2015. 491 p. 58-69.

[24] Mankarious, R.A., et al., Bulletproof vests/shields prepared from composite material based on strong polyamide fibers and epoxy resin. Journal of Engineering and Applied Sciences. 12(10): p. 2697-2701.

[25] Mohsen, W., M.A. Sadek, and H.A. Elazab, Green synthesis of copper oxide nanoparticles in aqueous medium as a potential efficient catalyst for catalysis applications. International Journal of Applied Engineering Research.12 (24): p. 14927-14930.

[26] Mostafa, A.R., H.A.-S. Omar, and A.E. Hany, Preparation of Hydrogel Based on Acryl Amide and Investigation of Different Factors Affecting Rate and Amount of Absorbed Water. Agricultural Sciences. Vol.08No.02: p. 11

[27] Radwan, M.A., et al., Mechanical characteristics for different composite materials based on commercial epoxy resins and different fillers. Journal of Engineering and Applied Sciences. 12(5): p. 1179-1185.

[28] Kobayashi, T., Kanematsu, H., Hashimoto, R., Morisato, K., Ohashi, N., Yamasaki, H., \& Takamiya, S. (2013). Study on Environment and Energy Using Belonging Materials. International Journal of Sustainable Development \& World Policy, 2(4), 50.

[29] Chan, Wu-Chung, and Hong-Siou Guo. "Preparation of a Poly (Vinylalcohol) (PVA)/Peat/Organoclay/Kno3 Composite Bead as Biofilter Material for Biofiltration of Volatile Organic Compounds." The International Journal of Biotechnology 3, no. 2 (2014): 24-31.

[30] Nwufo, B. T., Priscila, A. U., \& Onche, E. U. (2014). Production of Cost-Effective Adsorbents from Native Materials. International Journal of Chemistry and Materials Research, 2(4), 30-35.

[31] El-Agez, T. M., El-Ghamri, H. S., Abdel-Latif, M. S., Taya, S. A., \& Alkanoo, A. A. (2014). Thermoelectricity Based on Cuo as a Semiconducting Material. International Journal of Chemistry and Materials Research, 2(12), 166-173.

[32] Murugadoss, K., \& Pasupathi, G. (2015). Hardness Studies on Solution Grown Lithium Potassium Sulphate Single Crystals-An Inorganic Nonlinear Optical Material. International Journal of Chemistry and Materials Research, 3(1), 11-16.

[33] B. Ashraf, M. A. Radwan, M. A. Sadek, H. A. Elazab International Journal of Engineering and Technology(UAE), 7, 1295-1298.

[34] H. A. Elazab Biointerface Research in Applied Chemistry, 8, 3314 3318.

[35] H. A. Elazab Biointerface Research in Applied Chemistry, 8, 3278 3281.

[36] H. A. Elazab, M. A. Radwan, T. T. El-Idreesy International Journal of Nanoscience, 2018.

[37] H. A. Elazab, M. A. Sadek, T. T. El-Idreesy Adsorption Science \& Technology, 36, 1352-1365.

[38] N. S. Samir, M. A. Radwan, M. A. Sadek, H. A. Elazab International Journal of Engineering and Technology(UAE), 7, 1290-1294.

[39] Fatma Zakaria, M. A. Radwan, M. A. Sadek, H. A. Elazab International Journal of Engineering and Technology(UAE), 7, 2018.

[40] Reem Nasser, M. A. Radwan, M. A. Sadek, H. A. Elazab International Journal of Engineering and Technology(UAE), 7, 2018.

[41] Mostafa Ghobashy, Mamdouh Gadallah, Tamer T. El-Idreesy, M. A. Sadek, H. A. Elazab International Journal of Engineering and Technology(UAE), 7, 2018.

[42] H. A. Elazab, M. A. Sadek, Biointerface Research in Applied Chemistry, 8, 2018.

[43] Hany A. Elazab, The Canadian Journal of Chemical Engineering, volume 96 , issue 10, 2018. 